\title{
Association of polymorphisms in WWOX gene with risk and outcome of osteosarcoma in a sample of the young Chinese population
}

This article was published in the following Dove Press journal:

OncoTargets and Therapy

19 February 2016

Number of times this article has been viewed

\author{
Niannian Zhang' \\ Zhenghui Jiang ${ }^{2,3}$ \\ Weifeng Ren' \\ Li Yuan ${ }^{4}$ \\ Yangyi Zhu' \\ 'Department of Orthopedics, \\ Shaoxing Shangyu People's Hospital, \\ Shaoxing, People's Republic of \\ China; ${ }^{2}$ Division of Musculoskeletal \\ Oncology, Department of \\ Orthopedics, The Second Affiliated \\ Hospital of Zhejiang University School \\ of Medicine, Hangzhou, People's \\ Republic of China; ${ }^{3}$ Department \\ of Orthopedics, The First People's \\ Hospital of Wenling, Wenling, People's \\ Republic of China; ${ }^{4}$ Department of \\ Epidemiology, School of Public Health, \\ Fudan University, Shanghai, People's \\ Republic of China
}

\begin{abstract}
The WW domain-containing oxidoreductase $(W W O X)$ gene is a tumor suppressor gene, the abnormal expression of which will lead to osteosarcoma tumorigenesis. Polymorphisms of the $W W O X$ gene are associated with the risk of several malignancies. We hypothesized that genetic variations in the $W W O X$ gene were related to osteosarcoma risk and outcome. In this case-control study, we recruited 276 young osteosarcoma patients and 286 controls from the East Chinese population and genotyped seven tag single-nucleotide polymorphisms (SNPs) of the WWOX gene (rs10220974C $>$ T, rs $12918952 \mathrm{G}>\mathrm{A}, \mathrm{rs} 3764340 \mathrm{C}>\mathrm{G}, \mathrm{rs} 1074963 \mathrm{C}>\mathrm{G}$, $\mathrm{rs} 383362 \mathrm{G}>\mathrm{T}, \mathrm{rs} 1424110 \mathrm{~A}>\mathrm{G}$, and $\mathrm{rs} 12828 \mathrm{~A}>\mathrm{G})$. We discovered that two SNPs (rs3764340C $>\mathrm{G}$ and $\mathrm{rs} 383362 \mathrm{G}>\mathrm{T}$ ) were associated with osteosarcoma risk. The CG genotype and dominant model of rs3764340 indicated elevated risk of osteosarcoma, and similar results were found for rs383362. Furthermore, rs3754340C $>\mathrm{G}$ was also related to grade and metastasis risk of osteosarcoma. Taken together, our results provide the first evidence that $W W O X$ gene polymorphisms have the potential to be predictive factors for assessing risk and outcome of osteosarcoma.
\end{abstract}

Keywords: osteosarcoma, $W W O X$, SNP, metastasis, susceptibility

\section{Introduction}

Osteosarcoma is the most common musculoskeletal malignancy in childhood and adolescence, characterized by early metastatic potential to the lung and poor prognosis. ${ }^{1}$ Nonsurgical therapeutic regimens, which are essential for osteosarcoma treatment, consist of chemotherapies composed of four agents. However, some osteosarcoma patients suffer from chemoresistance and require more aggressive chemotherapy strategies. Furthermore, there are still only limited biomarkers (that can be easily detected in the laboratory) for predicting outcomes or malignancy risk.

Single-nucleotide polymorphisms (SNPs) are genetic factors that have been associated with cancer risk or progression. Studies on SNPs in osteosarcoma are valuable for identifying prognostic markers. Efforts have focused on the identification of reliable associations between polymorphisms in different genes and osteosarcoma risk, such as $E R C C,{ }^{2} V E G F,{ }^{3} N A T 2,{ }^{4}$ etc. However, studies on tumor suppressor gene polymorphisms in osteosarcoma are still limited.

The WW-domain containing oxidoreductase ( $W W O X)$ gene was demonstrated as a bona fide tumor suppressor gene, located on chromosome 16q23, spanning the common fragile site FRA16D. ${ }^{5}$ Evidence suggested that the $W W O X$ gene plays an important role in preventing osteosarcoma tumorigenesis, as $W W O X$ knockout mice showed significantly increased osteosarcoma susceptibility. ${ }^{6}$ Furthermore, in in vitro studies
Correspondence: Yangyi Zhu Department of Orthopedics, Shaoxing Shangyu People's Hospital, 517 Shimin Ave, Shaoxing, Zhejiang, People's Republic of China Tel +86 575 822I 358I Email zhuyangyisx@gmail.com (c) (1) (-) 2016 Zhang et al. This work is published and licensed by Dove Medical Press Limited. The full terms of this license are available at https://www.dovepress.com/terms.php cc) hereby accept the Terms. Non-commercial uses of the work are permitted without any further permission from Dove Medical Press Limited, provided the work is properly attributed. For permission for commercial use of this work, please see paragraphs 4.2 and 5 of our Terms (https://www.dovepress.com/terms.php). 
that increased expression of $W W O X$ via transfection in tumor cell lines, tumor suppression activities were demonstrated. ${ }^{7,8}$ In addition, it was recently discovered that normally expressed $W W O X$ inhibits osteosarcoma metastasis by modulating the function of some downstream proteins, ${ }^{9}$ and attenuation of the WWOX gene in osteosarcoma was associated with elevated tumorigenicity. ${ }^{10,11}$ Polymorphisms of the $W W O X$ gene were also found to be related to risk of incidence or outcome of some other solid tumors, such as glioma, ${ }^{12}$ lung cancer, ${ }^{13}$ and thyroid cancer. ${ }^{14}$ Given the fact that $W W O X$ plays a role in osteosarcoma, and that its gene polymorphisms were related to solid tumor risk, it is reasonable to hypothesize that $W W O X$ polymorphisms are involved in progression osteosarcoma and that $W W O X$ SNPs have potential as predicting factors.

In order to test the hypothesis that $W W O X$ polymorphisms are correlated with osteosarcoma risk or progression in young Chinese individuals, four hospitals and institutions located in East China participated in this project and shared clinical data. A case-control study was launched, and we analyzed seven WWOX tagging SNPs in 276 osteosarcoma patients and 286 controls from a young East China population.

\section{Material and methods Ethics approval}

This case-control study was approved by the Ethics Committees of the four participating institutions (Shaoxing Shangyu People's Hospital, The Second Affiliated Hospital of Zhejiang University School of Medicine, The First People's Hospital of Wenling, and Shanghai Fudan University). All investigations were performed according to the Declaration of Helsinki. Written informed consent to participate in genomic research was obtained beforehand from each participant or their guardians.

\section{Study participants}

A total of 276 newly diagnosed primary osteosarcoma patients under the age of 20 years and 286 cancer-free controls were recruited in this study. All patients were diagnosed on the basis of pathologic evidence, and samples were collected during the period from February 2007 to September 2012. All patients underwent appropriate surgical resections (including limb salvage and amputation) by qualified orthopedic surgeons, as well as nonsurgical therapeutic regimens according to the guidelines, and were followed up regularly for at least 36 months. All clinical information was obtained from medical records. Cancer-free controls were recruited from nonpathological fracture cases, and were matched to patient cases by age and sex. Blood samples were obtained from each participant, and tumor tissues were preserved in liquid nitrogen.

\section{DNA isolation}

Whole DNA was isolated from blood samples by standard phenol-chloroform extraction and ethanol precipitation. Genomic DNA was isolated using a DNA Blood Mini Kit (Qiagen, Berlin, Germany). For tumor tissues, proteinase K digestion was performed followed by phenol-chloroform extraction.

\section{SNP genotyping}

Seven SNPs $(W W O X$ rs10220974C $>$ T, rs12918952G $>$ A, rs $3764340 \mathrm{C}>\mathrm{G}, \operatorname{rs} 1074963 \mathrm{C}>\mathrm{G}, \operatorname{rs} 383362 \mathrm{G}>\mathrm{T}$, rs $1424110 \mathrm{~A}>\mathrm{G}$, and $\mathrm{rs} 12828 \mathrm{~A}>\mathrm{G}$ ) were selected for this study. Procedures were followed according to methods described in our previous study. ${ }^{4}$ Briefly, the Sequence Detection Software of the ABI StepOnePlus System (Thermo Fisher Scientific, Waltham, MA, USA) was used to collect data. TaqMan ${ }^{\circledR}$ primers and probes were designed by the ABI Assays-by-Design custom service. All tests were performed on samples in triplicate and repeated twice. Amplification conditions were $95^{\circ} \mathrm{C}$ for 10 minutes, followed by 40 cycles at $95^{\circ} \mathrm{C}$ for 15 seconds, and at $60^{\circ} \mathrm{C}$ for 60 seconds. Plates were read with the corresponding ABI Software.

\section{Haplotype analysis}

The computational haplotyping method was utilized in this study. The seven SNPs were analyzed on SHEsis (http:// analysis.bio-x.cn/myAnalysis.php) to identify frequent haplotypes (those with proportions over $3 \%$ ).

\section{Statistical analysis}

SPSS software (v21.0; IBM Corporation, Armonk, NY, USA) was used for statistical analysis. Similar to our previous research, statistical differences in distributions of selected variables, subject characteristics, and genotypes for $W W O X$ were evaluated between osteosarcoma cases and controls by $\chi^{2}$ tests. Odd ratios (ORs) and $95 \%$ confidence intervals ( $95 \%$ CIs) were calculated to test for correlations between the seven SNPs and the risk of osteosarcoma incidence. Logistic regression analyses were performed to calculate crude ORs, and ORs were subsequently adjusted for age and sex. The Hardy-Weinberg equilibrium for SNPs was tested using Pearson's $\chi^{2}$ test. Statistical significance was defined as $P<0.05$, and all analyses were two-sided.

\section{Results \\ Clinical features}

Clinical characteristics of the included samples are shown in Table 1. In this study, we recruited 276 osteosarcoma 
Table I General characteristics of the subjects

\begin{tabular}{|c|c|c|c|}
\hline Variables & $\begin{array}{l}\text { Osteosarcoma } \\
\text { cases, n (\%) }\end{array}$ & $\begin{array}{l}\text { Controls, } \\
\text { n (\%) }\end{array}$ & $P$-value \\
\hline Age (mean $\pm S D)$ & $16 \pm 3.28$ & $16.24 \pm 3.30$ & 0.378 \\
\hline \multicolumn{4}{|l|}{ Sex } \\
\hline Male & $162(58.70)$ & I 59 (55.59) & 0.458 \\
\hline Female & II 4 (4I.30) & I $27(44.4$ I) & \\
\hline \multicolumn{4}{|l|}{ Location } \\
\hline Trunk & $34(12.32)$ & & \\
\hline Limbs & $242(87.68)$ & & \\
\hline \multicolumn{4}{|l|}{ Enneking stages } \\
\hline IA or IB & 42 (I5.22) & & \\
\hline IIA or IIB or III & 234 (84.78) & & \\
\hline \multicolumn{4}{|l|}{ Operation } \\
\hline Amputation & $55(19.93)$ & & \\
\hline Limb salvage & $221(80.07)$ & & \\
\hline \multicolumn{4}{|l|}{ Metastasis } \\
\hline Yes & $53(19.20)$ & & \\
\hline No & $223(80.80)$ & & \\
\hline
\end{tabular}

Abbreviation: SD, standard deviation.

cases and 286 controls. There were 162 male and 114 female patients. The median ages and ranges of the osteosarcoma cases and controls were 16 years (6-20 years) and 16.24 years (5-20 years), respectively. Tumors were graded according to the Enneking GTM system. ${ }^{15}$ No statistical differences in age or sex were found between the two groups ( $P=0.378$ and 0.458 , respectively).

\section{Associations between WWOX polymorphisms and risk of osteosarcoma} The seven SNPs (rs10220974C $>$ T, rs12918952G $>$ A, rs $3764340 \mathrm{C}>\mathrm{G}, \operatorname{rs} 1074963 \mathrm{C}>\mathrm{G}, \mathrm{rs} 383362 \mathrm{G}>\mathrm{T}$, $\operatorname{rs} 1424110 \mathrm{~A}>\mathrm{G}$, and $\operatorname{rs} 12828 \mathrm{~A}>\mathrm{G}$ ) tested in this study are shown in Table 2. Genotype distributions of each of the seven SNPs were in Hardy-Weinberg equilibrium in the control group $(P=0.356,0.800,0.212,0.641,0.305,0.261$, and 0.152 , respectively). For $W W O X \operatorname{rs} 3764340 \mathrm{C}>\mathrm{G}$, single locus analyses showed no statistical difference in genotype between cases and controls. However, when the $\mathrm{CC}$ homozygote genotype was used as the reference group, the $\mathrm{CG}$ genotype showed significantly increased risk of osteosarcoma (CG vs CC: crude OR $=1.73$, 95\% CI $=1.15-2.61, P=0.009$; adjusted $\mathrm{OR}=1.74,95 \%$ $\mathrm{CI}=1.15-2.63, P=0.009)$. The GG genotype was not related to risk of osteosarcoma (although a borderline difference was found). In the dominant model, an even stronger statistical difference was detected (CG/GG vs CC: crude $\mathrm{OR}=1.82,95 \% \mathrm{CI}=1.23-2.69, P=0.003$; adjusted $\mathrm{OR}=1.82,95 \% \mathrm{CI}=1.22-2.70, P=0.003)$. In the recessive model, no statistically significant differences were found.
Similar to rs3764340C $>\mathrm{G}$, there was an association between the WWOX rs $383362 \mathrm{G}>\mathrm{T}$ SNP and osteosarcoma risk. The GT genotype was associated with an increased risk of osteosarcoma (GT vs GG: crude OR $=1.59$, 95\% CI $=1.07-2.35, P=0.022$; adjusted $\mathrm{OR}=1.56,95 \%$ $\mathrm{CI}=1.05-2.32, P=0.027)$. Although no correlation with osteosarcoma risk was found for the TT genotype, the dominant model (GT/TT) showed evidence for association with risk of osteosarcoma (GT/TT vs GG: crude OR $=1.60$, 95\% CI $=1.10-2.34, P=0.015$; adjusted $\mathrm{OR}=1.57,95 \%$ $\mathrm{CI}=1.07-2.30, P=0.020$ ).

No association for osteosarcoma susceptibility in the young Chinese population was found for the remaining five SNPs (rs10220974C $>$ T, rs12918952G $>$ A, rs $1074963 C>G$, rs $1424110 \mathrm{~A}>\mathrm{G}$, and $\operatorname{rs} 12828 \mathrm{~A}>\mathrm{G})$.

\section{Associations between WWOX polymorphisms and stage or metastasis of osteosarcoma}

Data on clinical features (location, surgical stage, operation method, and metastasis) of osteosarcoma cases were collected to reliably investigate the relationship between SNPs and incidence of osteosarcoma (Table 3). For WWOX rs3764340C $>\mathrm{G}$, the frequency of the genotype $\mathrm{CG}$ at late Enneking stages (28.63\%) was greater when compared with early-stage cases $(9.52 \%)$, and a statistical difference in frequency distribution was found $(P=0.033)$. Moreover, a similar result was shown for metastasis. The genotype $\mathrm{CG}$ displayed higher frequency $(32.08 \%)$ in metastatic cases compared with metastasis-free cases (24.22\%), and the difference in frequency distribution was statistically significant $(P=0.027)$.

For $W W O X$ rs $383362 \mathrm{G}>\mathrm{T}$, however, no correlation was found between the rs383362 SNP and clinical characteristics. Although the rs $383362 \mathrm{G}>\mathrm{T}$ SNP was found to be related to osteosarcoma susceptibility, it was not associated with osteosarcoma location $(P=0.357)$, stage $(P=0.939)$, operation method $(P=0.141)$, or metastasis $(P=0.539)$ (data shown in Table 4). The confounding variables are listed in Tables 5 and 6.

\section{Haplotype analyses showed statistical differences between cases and controls}

Online analyses of seven SNPs revealed eight frequent haplotypes (frequency over 3\%): CGCCGAG, CGCGGAA, CGCGGAG, CGCGGGA, CGCGGGG, CGCGTGG, CGGGAG, andCGGGGG(Table 7). Among them, CGCGGAG and CGCGTGG showed statistically significant differences 
Table 2 Logistic regression analyses of correlations between WWOX rs I0220974C $>$ T, rs |29|8952G $>$ A, rs $3764340 C>$ G, rs I074963C > G, rs383362G $>$ T, rs I424II0A $>$ G, and rs I2828A $>$ G polymorphisms and risk of osteosarcoma

\begin{tabular}{|c|c|c|c|c|c|c|c|c|}
\hline \multirow[t]{2}{*}{$\begin{array}{l}\text { WWOX } \\
\text { genotype }\end{array}$} & \multicolumn{2}{|c|}{$\begin{array}{l}\text { Cases } \\
(\mathrm{N}=276)\end{array}$} & \multicolumn{2}{|c|}{$\begin{array}{l}\text { Controls } \\
(\mathrm{N}=286)\end{array}$} & \multirow[t]{2}{*}{$\begin{array}{l}\text { Crude OR } \\
(95 \% \mathrm{Cl})\end{array}$} & \multirow[t]{2}{*}{$P$-value } & \multirow[t]{2}{*}{$\begin{array}{l}\text { Adjusted OR } \\
(95 \% \mathrm{Cl})\end{array}$} & \multirow[t]{2}{*}{$P$-value } \\
\hline & $\mathbf{n}$ & $\%$ & $\mathbf{n}$ & $\%$ & & & & \\
\hline \multicolumn{9}{|c|}{ WWOX rsI0220974C > T } \\
\hline $\mathrm{CC}$ & 211 & 76.45 & 228 & 79.72 & 1.00 & & 1.00 & \\
\hline $\mathrm{CT}$ & 61 & 22.10 & 53 & 18.53 & $1.24(0.82-1.88)$ & 0.301 & $1.24(0.82-1.88)$ & 0.308 \\
\hline $\mathrm{TT}$ & 4 & 1.45 & 5 & 1.75 & $0.86(0.23-3.26)$ & 0.830 & $0.85(0.23-3.22)$ & 0.812 \\
\hline $\mathrm{CT}+\mathrm{TT}$ & 65 & 23.55 & 58 & 20.28 & $\mathrm{I} .2 \mathrm{I}(0.8 \mathrm{I}-\mathrm{I} .8 \mathrm{I})$ & 0.349 & $1.21(0.81-1.80)$ & 0.360 \\
\hline $\mathrm{CC}+\mathrm{CT}$ & 272 & 98.55 & 281 & 98.25 & 1.00 & & 1.00 & \\
\hline TT & 4 & 1.45 & 5 & 1.75 & $0.83(0.22-3.11)$ & 0.78 & $0.81(0.22-3.07)$ & 0.76 \\
\hline \multicolumn{9}{|c|}{ WWOX rs I29/8952G $>A$} \\
\hline GG & 251 & 90.94 & 257 & 89.86 & 1.00 & & 1.00 & \\
\hline GA & 23 & 8.33 & 28 & 9.79 & $0.84(0.47-1.50)$ & 0.557 & $0.83(0.46-1.48)$ & 0.520 \\
\hline AA & 2 & 0.72 & 1 & 0.35 & $2.05(0.19-22.73)$ & 0.559 & $2.25(0.20-25.13)$ & 0.509 \\
\hline $\mathrm{GA}+\mathrm{AA}$ & 25 & 9.05 & 29 & 10.14 & $0.88(0.50-1.55)$ & 0.664 & $0.87(0.50-1.54)$ & 0.638 \\
\hline $\mathrm{GG}+\mathrm{GA}$ & 274 & 99.28 & 285 & 99.65 & 1.00 & & 1.00 & \\
\hline AA & 2 & 0.72 & I & 0.35 & $2.08(0.19-23.07)$ & 0.551 & $2.29(0.2 \mathrm{I}-25.5 \mathrm{I})$ & 0.501 \\
\hline \multicolumn{9}{|c|}{ WWOX rs $3764340 C>G$} \\
\hline $\mathrm{CC}$ & 194 & 70.29 & 232 & 81.12 & 1.00 & & 1.00 & \\
\hline CG & 71 & 25.72 & 49 & 17.13 & $1.73(1.15-2.61)$ & $0.009 *$ & $1.74(1.15-2.63)$ & $0.009 *$ \\
\hline GG & II & 3.99 & 5 & 1.75 & $2.63(0.90-7.70)$ & 0.078 & $2.57(0.88-7.56)$ & 0.086 \\
\hline $\mathrm{CG}+\mathrm{GG}$ & 82 & 29.71 & 54 & 18.88 & $1.82(1.23-2.69)$ & $0.003^{*}$ & $1.82(1.22-2.70)$ & $0.003^{*}$ \\
\hline $\mathrm{CC}+\mathrm{CG}$ & 265 & 96.01 & 281 & 98.25 & 1.00 & & 1.00 & \\
\hline GG & II & 3.99 & 5 & 1.75 & $2.33(0.80-6.80)$ & 0.121 & $2.27(0.78-6.63)$ & 0.135 \\
\hline \multicolumn{9}{|c|}{ WWOX rs $1074963 C>G$} \\
\hline $\mathrm{CC}$ & 4 & 1.45 & 7 & 2.45 & 1.00 & & 1.00 & \\
\hline CG & 72 & 26.09 & 69 & 24.13 & $1.83(0.5 \mathrm{I}-6.52)$ & 0.354 & $1.93(0.54-6.93)$ & 0.312 \\
\hline GG & 200 & 72.46 & 210 & 73.43 & $1.67(0.48-5.78)$ & 0.421 & $1.79(0.5 \mathrm{I}-6.24)$ & 0.363 \\
\hline $\mathrm{CG}+\mathrm{GG}$ & 272 & 98.55 & 279 & 97.55 & I.7I (0.49-5.89) & 0.398 & $1.83(0.53-6.34)$ & 0.344 \\
\hline$C C+C G$ & 76 & 27.54 & 76 & 26.58 & 1.00 & & 1.00 & \\
\hline GG & 200 & 72.46 & 210 & 73.43 & $0.95(0.66-1.38)$ & 0.797 & $0.97(0.67-I .4 I)$ & 0.862 \\
\hline \multicolumn{9}{|c|}{ WWOX rs383362G $>T$} \\
\hline GG & 190 & 68.84 & 223 & 77.97 & 1.00 & & 1.00 & \\
\hline GT & 77 & 27.90 & 57 & 19.93 & $1.59(1.07-2.35)$ & $0.022^{*}$ & $1.56(1.05-2.32)$ & $0.027^{*}$ \\
\hline TT & 9 & 3.26 & 6 & 2.10 & $1.76(0.62-5.04)$ & 0.292 & $1.65(0.57-4.77)$ & 0.354 \\
\hline $\mathrm{GT}+\mathrm{TT}$ & 86 & 31.16 & 63 & 22.03 & $1.60(1.10-2.34)$ & $0.015^{*}$ & $1.57(1.07-2.30)$ & $0.020 *$ \\
\hline $\mathrm{GG}+\mathrm{GT}$ & 267 & 96.74 & 280 & 97.90 & 1.00 & & 1.00 & \\
\hline TT & 9 & 3.26 & 6 & 2.10 & $1.57(0.55-4.48)$ & 0.396 & $\mathrm{I} .46(0.5 \mid-4.2 \mathrm{I})$ & 0.480 \\
\hline \multicolumn{9}{|c|}{ WWOX rs $1424 I I O A>G$} \\
\hline $\mathrm{AA}$ & 88 & 31.88 & 108 & 37.76 & 1.00 & & 1.00 & \\
\hline AG & 136 & 49.28 & 127 & 44.41 & 1.31 (0.9|-I.9I) & 0.149 & $1.31(0.90-1.90)$ & 0.154 \\
\hline GG & 52 & 18.84 & 51 & 17.83 & $1.25(0.78-2.02)$ & 0.358 & $1.25(0.78-2.02)$ & 0.356 \\
\hline$A G+G G$ & 188 & 68.12 & 178 & 62.24 & $1.30(0.92-1.84)$ & 0.144 & $1.29(0.9|-| .83)$ & 0.148 \\
\hline$A A+A G$ & 224 & 81.16 & 235 & 82.17 & 1.00 & & 1.00 & \\
\hline GG & 52 & 18.84 & 51 & 17.83 & $1.07(0.70-1.64)$ & 0.757 & $1.07(0.70-1.65)$ & 0.747 \\
\hline \multicolumn{9}{|c|}{ WWOX $\mathrm{rs} \mid 2828 \mathrm{~A}>\mathrm{G}$} \\
\hline $\mathrm{AA}$ & 20 & 7.25 & 24 & 8.39 & 1.00 & & 1.00 & \\
\hline AG & 125 & 45.29 & 135 & 47.20 & I.II (0.59-2.II) & 0.747 & $1.09(0.57-2.08)$ & 0.796 \\
\hline GG & $13 \mid$ & 47.46 & 127 & $44.4 I$ & $1.24(0.65-2.35)$ & 0.515 & $1.21(0.63-2.30)$ & 0.570 \\
\hline$A G+G G$ & 256 & 92.75 & 262 & 91.61 & $1.17(0.63-2.18)$ & 0.614 & $1.15(0.62-2.13)$ & 0.668 \\
\hline$A A+A G$ & 145 & 52.54 & 159 & 55.59 & 1.00 & & 1.00 & \\
\hline GG & $13 \mid$ & 47.46 & 127 & $44.4 I$ & $1.13(0.8 \mathrm{I}-1.58)$ & 0.467 & $1.12(0.80-1.56)$ & 0.502 \\
\hline
\end{tabular}

Notes: ORs were adjusted for age and sex. *Statistically significant $(P<0.05)$.

Abbreviations: $\mathrm{OR}$, odds ratio; $\mathrm{Cl}$, confidence interval. 
Table 3 Association between genotype frequencies of WWOX rs3764340 and clinical features in osteosarcoma cases

\begin{tabular}{|c|c|c|c|c|c|}
\hline Variables & $\mathbf{n}$ & CC, n (\%) & CG, n (\%) & GG, n (\%) & $P$-value \\
\hline \multicolumn{6}{|l|}{ Location } \\
\hline Trunk & 34 & $26(76.47)$ & $5(14.7 \mid)$ & $3(8.82)$ & 0.115 \\
\hline Limbs & 242 & $168(69.42)$ & $66(27.27)$ & $8(3.31)$ & \\
\hline \multicolumn{6}{|l|}{ Enneking stages } \\
\hline IA or IB & 42 & 36 (85.7I) & $4(9.52)$ & $2(4.76)$ & $0.033^{*}$ \\
\hline IIA or IIB or III & 234 & I 58 (67.52) & $67(28.63)$ & $9(3.85)$ & \\
\hline \multicolumn{6}{|l|}{ Operation } \\
\hline Amputation & 55 & $42(76.36)$ & $10(18.18)$ & $3(5.45)$ & 0.324 \\
\hline Limb salvage & 221 & $152(68.78)$ & $61(27.60)$ & $8(3.62)$ & \\
\hline \multicolumn{6}{|l|}{ Metastasis } \\
\hline Yes & 53 & 31 (58.49) & $17(32.08)$ & $5(9.43)$ & $0.027^{*}$ \\
\hline No & 223 & $163(73.08)$ & $54(24.22)$ & $6(2.69)$ & \\
\hline
\end{tabular}

Note: *Statistically significant $(P<0.05)$.

between cases and controls $(P<0.001,95 \% \mathrm{CI}=0.41-0.75$; $P=0.005,95 \% \mathrm{CI}=1.30-5.23$, respectively).

\section{Discussion}

Studies on new therapeutic strategies against osteosarcoma are emerging, especially for different kinds of immunotherapy, which are showing promising results. ${ }^{16-18}$ However, prediction factors, especially those that can be easily detected in laboratory assays, remain limited. Recently, several studies have revealed potential factors that may be utilized to predict osteosarcoma outcome or metastasis risk, ${ }^{19,20}$ but studies based on clinical data have still been limited (or have sometimes had controversial results). Given that osteosarcoma is not a common malignancy, we collected blood samples and tumor tissues of osteosarcoma patients from different hospitals located in East China (which has a stable population of over 100 million) and tried to identify indicators predictive of disease risk. In our previous study,

Table 4 Association between genotype frequencies of WWOX rs383362 and clinical features in osteosarcoma cases

\begin{tabular}{|c|c|c|c|c|c|}
\hline Variables & $\mathbf{n}$ & $\begin{array}{l}\text { GG } \\
\text { n (\%) }\end{array}$ & $\begin{array}{l}\text { GT } \\
\text { n (\%) }\end{array}$ & $\begin{array}{l}\text { TT } \\
\text { n (\%) }\end{array}$ & $P$-value \\
\hline \multicolumn{6}{|l|}{ Location } \\
\hline Trunk & 34 & $20(58.8)$ & $13(38.24)$ & I (2.94) & 0.357 \\
\hline Limbs & 242 & $170(70.25)$ & $64(26.45)$ & $8(3.31)$ & \\
\hline \multicolumn{6}{|l|}{ Enneking stages } \\
\hline IA or IB & 42 & $29(69.05)$ & $12(28.57)$ & I (2.38) & 0.939 \\
\hline IIA or IIB or III & 234 & I6I (68.80) & 65 (27.78) & $9(3.42)$ & \\
\hline \multicolumn{6}{|l|}{ Operation } \\
\hline Amputation & 55 & $32(58.18)$ & $20(36.36)$ & $3(5.45)$ & 0.141 \\
\hline Limb salvage & 221 & I 58 (7|.49) & 57 (25.79) & $6(2.7 \mathrm{I})$ & \\
\hline \multicolumn{6}{|l|}{ Metastasis } \\
\hline Yes & 53 & $35(66.04)$ & I5 (28.30) & $3(5.66)$ & 0.539 \\
\hline No & 223 & I55 (69.5I) & $62(27.80)$ & $6(2.69)$ & \\
\hline
\end{tabular}

Table 5 Confounding variables (Enneking stages)

\begin{tabular}{llll}
\hline Confounding variables & $\begin{array}{l}\text { IA or IB } \\
\text { cases, } \mathbf{n}(\%)\end{array}$ & $\begin{array}{l}\text { IIA or IIB or } \\
\text { III cases, } \mathbf{n}(\%)\end{array}$ & P-value \\
\hline $\begin{array}{l}\text { Age, mean } \pm \text { SD (years) } \\
\text { Sex }\end{array}$ & $16.50 \pm 2.84$ & $15.9 I \pm 3.35$ & 0.284 \\
$\quad$ Male & $21(50)$ & $14 \mid(60.26)$ & 0.214 \\
$\quad$ Female & $21(50)$ & $93(39.74)$ & \\
\hline
\end{tabular}

Abbreviation: SD, standard deviation.

our colleagues found that NAT2 gene polymorphisms were associated with osteosarcoma risk, ${ }^{4}$ but the reason for the effects of NAT2 polymorphisms on osteosarcoma risk and outcome has remained unclear. We therefore continued to search for genes involved in osteosarcoma pathogenesis using our blood and tissue sample bank.

Currently, clinicians usually make therapeutic plans for osteosarcomas based on imaging data and clinical symptoms, while laboratory examinations are seldom used. If effective genotype analyses could be established, clinicians would have more evidence for making decisions on more radical therapeutic regimens for high-risk patients. In this casecontrol study, we discovered that $W W O X$ rs3764340C $>\mathrm{G}$ and $\mathrm{rs} 383362 \mathrm{G}>\mathrm{T}$ SNPs were associated with increased risk of osteosarcoma. Furthermore, to determine if $W W O X$ SNPs are associated with outcomes of osteosarcoma, we analyzed SNPs with clinical data, including age, sex, location, grade, operation method, and metastasis. Here, we demonstrated that $\mathrm{rs} 3764340 \mathrm{C}>\mathrm{G}$ was related to the grade of osteosarcoma and metastasis. Moreover, although rs383362G $>\mathrm{T}$ was found to be associated with osteosarcoma risk, no correlation was found in grade or metastasis risk. As $W W O X$ is an established tumor suppressor gene, our results, indicating that polymorphisms in this gene are related to osteosarcoma, seem reasonable. We therefore believe that people with particular WWOX genotypes will have corresponding osteosarcoma risks and outcomes.

In addition to SNP analyses, haplotyping was performed. The haplotype CGCGGAG was more frequent in normal individuals, while CGCGTGG (which replaced $\mathrm{G}$ with $\mathrm{T}$ ) was

Table 6 Confounding variables (metastasis)

\begin{tabular}{llll}
\hline Confounding variables & $\begin{array}{l}\text { Metastasis } \\
\text { cases, } \mathbf{n}(\%)\end{array}$ & $\begin{array}{l}\text { Nonmetastasis } \\
\text { cases, n (\%) }\end{array}$ & P-value \\
\hline $\begin{array}{l}\text { Age, mean } \pm \text { SD (years) } \\
\text { Sex }\end{array}$ & $15.72 \pm 3.57$ & $16.07 \pm 3.21$ & 0.485 \\
$\quad$ Male & $34(64.15)$ & $128(57.40)$ & 0.370 \\
$\quad$ Female & $19(35.85)$ & $95(42.60)$ & \\
\hline
\end{tabular}

Abbreviation: SD, standard deviation. 
Table 7 Haplotype analysis

\begin{tabular}{|c|c|c|c|c|}
\hline \multirow[t]{2}{*}{ Haplotype } & $\begin{array}{l}\text { Cases } \\
(\mathrm{N}=276)\end{array}$ & $\begin{array}{l}\text { Controls } \\
(\mathrm{N}=286)\end{array}$ & \multirow[t]{2}{*}{$P$-value } & \multirow[t]{2}{*}{ OR $(95 \% \mathrm{Cl})$} \\
\hline & quency) & iency) & & \\
\hline CGCCGAG & $16.55(0.03)$ & $24.22(0.04)$ & 0.236 & $0.68(0.36-1.29)$ \\
\hline CGCGGAA & $56.81(0.10)$ & $56.60(0.01)$ & 0.917 & $1.02(0.69-1.52)$ \\
\hline CGCGGAG & $105.19(0.19)$ & $159.12(0.28)$ & $<0.00 I^{*}$ & $0.56(0.4 I-0.75)$ \\
\hline CGCGGGA & $41.18(0.08)$ & $47.53(0.08)$ & 0.522 & $0.87(0.56-1.35)$ \\
\hline CGCGGGG & $66.81(0.12)$ & $89.56(0.16)$ & 0.055 & $0.7 \mathrm{I}(0.50-1.0 \mathrm{I})$ \\
\hline CGCGTGG & $28.45(0.05)$ & I I.58 (0.02) & $0.005^{*}$ & $2.60(1.30-5.23)$ \\
\hline CGGGGAG & $20.08(0.04)$ & $11.09(0.02)$ & 0.092 & $1.88(0.89-3.97)$ \\
\hline CGGGGGG & $18.96(0.03)$ & $10.09(0.02)$ & 0.086 & $1.95(0.90-4.24)$ \\
\hline
\end{tabular}

Note: *Statistically significant $(P<0.05)$.

Abbreviations: $\mathrm{OR}$, odds ratio; $\mathrm{Cl}$, confidence interval.

more common in osteosarcoma patients. This result provides evidence that $\mathrm{rs} 383362 \mathrm{G}>\mathrm{T}$ plays a role in osteosarcoma.

Other studies on $W W O X$ rs3764340 revealed similar results. The CG/GG genotype of rs3764340 was accompanied by elevated risk of gastric cardia adenocarcinoma, ${ }^{21}$ lung cancer, ${ }^{13}$ and thyroid carcinoma, ${ }^{14}$ consistent with our results. Thus, we propose that carriers of the G allele of $W W O X$ rs3764340 may have increased cancer risk.

There are some limitations to our study. First, all blood or tumor tissue samples were collected from hospitals and, consequently, inherent bias might have affected our results. Second, due to the low morbidity of osteosarcoma, the sample size was limited, especially for homozygotic cases, which limits the statistical power. Third, as over half the recruited patients were still alive, we were not able to provide data analysis on survival. We will continue collecting samples and tracking clinical data to provide updated research results. Indeed, given the low morbidity of osteosarcoma (as low as around 5 per million), a prospective study would not be easy to implement and, consequently, we have no plans for such a study at present.

In summary, this study demonstrates that $W W O X \mathrm{rs} 3764340$ and rs383362 polymorphisms are associated with osteosarcoma susceptibility in young Chinese individuals. The WWOX rs3764340 polymorphism was thus correlated with surgical grade and metastatic risk of osteosarcoma.

\section{Disclosure}

The authors report no conflicts of interest in this work.

\section{References}

1. Picci P. Osteosarcoma (osteogenic sarcoma). Orphanet J Rare Dis. 2007;2:6.

2. Li J, Liu S, Wang W, et al. ERCC polymorphisms and prognosis of patients with osteosarcoma. Tumour Biol. 2014;35:10129-10136.
3. Wang Z, Wen P, Luo X, et al. Association of the vascular endothelial growth factor (VEGF) gene single-nucleotide polymorphisms with osteosarcoma susceptibility in a Chinese population. Tumour Biol. 2014;35: 3605-3610.

4. Huang Z, Yuan L, Jiang Z, Wang D. Associations of polymorphisms in NAT2 gene with risk and metastasis of osteosarcoma in young Chinese population. Onco Targets Ther. 2015;8:2675-2680.

5. Abu-Odeh M, Salah Z, Herbel C, Hofmann TG, Aqeilan RI. WWOX, the common fragile site FRA16D gene product, regulates ATM activation and the DNA damage response. Proc Natl Acad Sci U S A. 2014; 111:E4716-E4725.

6. Aqeilan RI, Trapasso F, Hussain S, et al. Targeted deletion of Wwox reveals a tumor suppressor function. Proc Natl Acad Sci U S A. 2007; 104:3949-3954.

7. Fabbri M, Iliopoulos D, Trapasso F, et al. WWOX gene restoration prevents lung cancer growth in vitro and in vivo. Proc Natl Acad Sci US A. 2005;102:15611-15616.

8. Gourley C, Paige AJ, Taylor KJ, et al. WWOX gene expression abolishes ovarian cancer tumorigenicity in vivo and decreases attachment to fibronectin via integrin $\alpha 3$. Cancer Res. 2009;69:4835-4842.

9. Del MS, Aqeilan RI. Tumor suppressor WWOX inhibits osteosarcoma metastasis by modulating RUNX2 function. Sci Rep. 2015;5:12959.

10. Kurek KC, Del MS, Salah Z, et al. Frequent attenuation of the WWOX tumor suppressor in osteosarcoma is associated with increased tumorigenicity and aberrant RUNX2 expression. Cancer Res. 2010;70: $5577-5586$.

11. Abdeen SK, Del MS, Hussain S, et al. Conditional inactivation of the mouse Wwox tumor suppressor gene recapitulates the null phenotype. J Cell Physiol. 2013;228:1377-1382.

12. Yu K, Fan J, Ding X, et al. Association study of a functional copy number variation in the WWOX gene with risk of gliomas among Chinese people. Int J Cancer. 2014;135:1687-1691.

13. Huang D, Qiu F, Yang L, et al. The polymorphisms and haplotypes of WWOX gene are associated with the risk of lung cancer in southern and eastern Chinese populations. Mol Carcinog. 2013;52(Suppl 1): E19-E27.

14. Cancemi L, Romei C, Bertocchi S, et al. Evidences that the polymorphism Pro-282-Ala within the tumor suppressor gene WWOX is a new risk factor for differentiated thyroid carcinoma. Int J Cancer. 2011;129: 2816-2824.

15. Enneking WF. A system of staging musculoskeletal neoplasms. Clin Orthop Relat Res. 1986:9-24.

16. Li B, Zhu X, Sun L, et al. Induction of a specific $\mathrm{CD} 8^{+} \mathrm{T}$-cell response to cancer/testis antigens by demethylating pre-treatment against osteosarcoma. Oncotarget. 2014;5:10791-10802.

17. Krishnadas DK, Shusterman S, Bai F, et al. A phase I trial combining decitabine/dendritic cell vaccine targeting MAGE-A1, MAGE-A3 and NY-ESO-1 for children with relapsed or therapy-refractory neuroblastoma and sarcoma. Cancer Immunol Immunother. 2015;64: 1251-1260.

18. Ahmed N, Brawley VS, Hegde M, et al. Human epidermal growth factor receptor 2 (HER2)-specific chimeric antigen receptor-modified T cells for the immunotherapy of HER2-positive sarcoma. J Clin Oncol. 2015;33: $1688-1696$.

19. Chen Y, Guo Y, Yang H, et al. TRIM66 overexpresssion contributes to osteosarcoma carcinogenesis and indicates poor survival outcome. Oncotarget. 2015;6:23708-23719.

20. Gemoll T, Epping F, Heinrich L, et al. Increased cathepsin D protein expression is a biomarker for osteosarcomas, pulmonary metastases and other bone malignancies. Oncotarget. 2015;6:16517-16526.

21. Guo W, Dong Z, Dong Y, Guo Y, Kuang G, Yang Z. Genetic and epigenetic alterations of WWOX in the development of gastric cardia adenocarcinoma. Environ Mol Mutagen. 2013;54:112-123. 
OncoTargets and Therapy

\section{Publish your work in this journal}

OncoTargets and Therapy is an international, peer-reviewed, open access journal focusing on the pathological basis of all cancers, potential targets for therapy and treatment protocols employed to improve the management of cancer patients. The journal also focuses on the impact of management programs and new therapeutic agents and protocols on

patient perspectives such as quality of life, adherence and satisfaction. The manuscript management system is completely online and includes a very quick and fair peer-review system, which is all easy to use. Visit http://www.dovepress.com/testimonials.php to read real quotes from published authors.

Submit your manuscript here: http://www.dovepress.com/oncotargets-and-therapy-journal 